(C2008 IEEE. Personal use of this material is permitted. However, permission to reprint/republish this material for advertising or promotional purposes or for creating new collective works for resale or redistribution to servers or lists, or to reuse any copyrighted component of this work in other works must be obtained from the IEEE 


\section{Guest Editorial \\ Introduction to the Special Section on M-Health: Beyond Seamless Mobility and Global Wireless Health-Care Connectivity}

\begin{abstract}
M-Health can be defined as "mobile computing, medical sensor, and communications technologies for health-care." This emerging concept represents the evolution of e-health systems from traditional desktop "telemedicine" platforms to wireless and mobile configurations. Current and emerging developments in wireless communications integrated with developments in pervasive and wearable technologies will have a radical impact on future health-care delivery systems. This editorial paper presents a snapshot of recent developments in these areas and addresses some of the challenges and future implementation issues from the $\mathrm{m}$-Health perspective. The contributions presented in this special section represent some of these recent developments and illustrate the multidisciplinary nature of this important and emerging concept.
\end{abstract}

Index Terms-Body area networks, m-Health, mobile technologies, sensors, wireless technologies.

\section{INTRODUCTION}

$\mathbf{T}$ HE TERM m-Health was first introduced implicitly as "Unwired e-med" in the first special issue of these transactions on wireless telemedicine systems [1]. Since then, there have been significant advances in wireless communications and network technologies with parallel advances in pervasive and wearable systems [2], [3]. These advances have already made a significant impact on current e-health and telemedical systems. In general terms, m-Health can be defined as "mobile computing, medical sensor, and communications technologies for health care."

The increased availability, miniaturization, performance, enhanced data rates, and the expected convergence of future wireless communication and network technologies around mobile health systems will accelerate the deployment of m-Health systems and services within the next decade. These will have a powerful impact on some of the existing health-care services and will reshape some of the mechanisms of existing health-care delivery routes. For example, development of smart intelligent sensors and drug delivery devices, some of them implanted, will allow communication with a personal server in complete mobility [4]. The personal server provides global connectivity to the telemedical server using a wireless personal area network (WPAN), wireless local area network (WLAN), or wireless wide area network (WAN). Developments in these areas are mainly driven by the evolving mass markets for cell phones and

Digital Object Identifier 10.1109/TITB.2004.840019 portable computing devices and represent an evolution of the previous generation of telemedical systems [1].

Traditionally, the "wireless concept" is associated closely with "biomonitoring." These have been used extensively in the last two decades to perform different data acquisition tasks mostly, without timely integration of data into the medical record; thus, no immediate action occurs if abnormalities are detected. Typical examples are Holter monitors that are routinely used for electrocardiogram (ECG) and electroencephalogram (EEG) monitoring.

Historically, "wireless monitoring" includes physiological monitoring of parameters such as heart rate, blood pressure, blood oximetry, and other physiological signals. Other areas include physical activity monitoring of parameters such as monitoring of movement, fall detection, location tracking, gastrointestinal telemetry, and other physical activities [5]. The benefits of the wireless technology have been illustrated in a number of different examples and applications [6].

Today with wireless technology, patient records could be accessed by health-care professionals from any given location by connection to the institution's information system. Physicians' access to patient history, laboratory results, pharmaceutical data, insurance information, and medical resources would be enhanced by mobile technology, thereby improving the quality of patient care. Handheld devices can also be used in home health care, for example, to fight diabetes through effective monitoring. A comprehensive overview of some of these existing wireless telemedicine applications and research can be found in recent publications in the area [1]-[3], [5], [6].

However, there are some limitations to existing wireless technologies that mostly depend on general packet radio service (GPRS) technologies and on their deployment strategies in health care. Some of these issues can be summarized as follows [7]:

1) The lack of an existing flexible and integrated "m-Health-on-demand" linkage of the different mobile telecommunication options and standards for e-Health services. This lack of linkage and compatibility for telemedical services exists due to the difficulty of achieving operational compatibility between the telecommunication services, terminals and devices standards, and "m-Health protocols."

2) The high cost of communication links, especially between satellites and global mobile devices and the limitation of existing wireless data rates especially for the globally available $2.5 \mathrm{G}$ and third-generation $(3 \mathrm{G})$ services for 


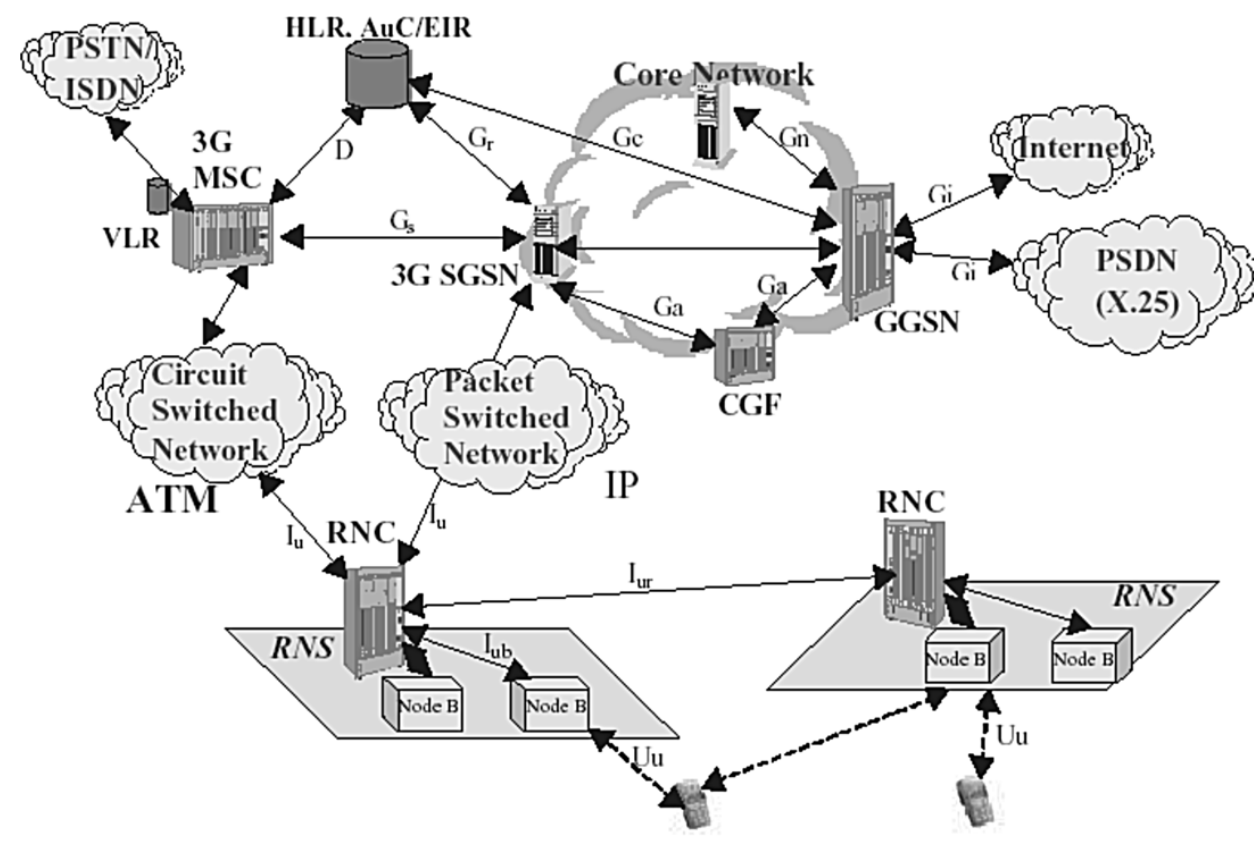

Fig. 1. General UMTS architecture.

some e-Health services. This is also combined with the availability of secure mobile Internet connectivity and information access especially for e-health systems.

3) Health-care is a very complex industry that is difficult to change. Organizational changes are very often required for health-care institutions to benefit from e-Health and m-Health services.

4) The short-term and long-term economic consequences and working conditions for physicians and health-care experts using these technologies are not yet fully understood or properly investigated.

5) The methods of payment and reimbursement issues for e-Health and m-Health services are not yet fully developed and standardized.

6) There is a lack of integration between existing e-Health services and other information systems, e.g., referral and ordering systems, medical records, etc.

7) The demonstration projects so far have failed to show that $\mathrm{m}$-Health services result in real savings and have costeffective potential.

These represent some of the factors that have hindered the wider applications of $\mathrm{m}$-Health technologies thus far across health-care systems. However, it is hoped that the current deployment of universal mobile telecommunications system (UMTS) networks globally will alleviate some of these issues and will provide a better and more effective platform for mobile health-care services.

\section{EMERGing m-HeAlth TeChNOLOGIES}

In this section, we highlight some of the more recent developments in these areas, present some of the evolving concepts, and discuss how these emerging technologies can meet the growing demand for future m-Health services.

\section{A. Impact of Future Wireless Communications and Mobile Networks on m-Health}

1) $3 G$ and Beyond Networking: The evolution of current $3 \mathrm{G}$ wireless communication and mobile network technologies will be the major driving force for future developments in $\mathrm{m}$-Health systems. 3G wireless technology represents the convergence of various second-generation wireless systems. One of the most important aspects of $3 \mathrm{G}$ technology is its ability to unify existing cellular standards, such as code-division multiple-access, global system for mobile communications (GSM), and time-division multiple-access, under one umbrella.

Fig. 1 shows the general architecture of a UMTS network [8]. The detailed description of these systems can be found in several recent UMTS texts so only a brief description of the basic architecture is presented here for completeness. In general, the UMTS architecture is divided into three major parts [8], [9]: the air interface, the UMTS Terrestrial Radio Access Network (UTRAN), and the Core Network. The latest commercial release of this system is R5. The Radio Network Controller (RNC) can be considered to be roughly the equivalent of the Base Station Controller in GSM and the Node Bs equate approximately to the GSM base stations (Base Transceiver Stations).

The RNCs and the base stations are collectively known as the UTRAN. From the UTRAN to the Core, the network is divided into packet and circuit-switched parts, the interface between the radio access and the core network $\left(l_{u}\right)$ being really two air interfaces: $l_{u}$ (PS- Packet Switch) and $l_{u}$ (CS-Circuit Switched). Packet traffic is concentrated in a new switching element - the Serving GPRS Support Node (SGSN). The boundary of the UMTS core network for packets is Gateway GPRS Support Node (GGSN), which is very much like a normal Internet protocol (IP) gateway and connects to corporate Intranets and the Internet.

2) Other Advances in Mobile Networks: In recent years, other mobile network technologies such as WLAN and WPANs 
have become popular [10]. These technologies are implemented as an extension to or as an alternative for wired LAN to make the communication more flexible and powerful.

WLAN allows users to access a data network at high speeds of up to $54 \mathrm{Mb} / \mathrm{s}$ as long as users are located within a relatively short range (typically $30-50 \mathrm{~m}$ indoors and $100-500 \mathrm{~m}$ outdoors) of a WLAN base station (or antenna). In the U.S., WLAN operates in two unlicensed bands [11], [12]:

a) $802.11 \mathrm{~b}$ and $802.11 \mathrm{~g}$ operate in the $2.4 \mathrm{GHz}$ band, together with many other devices including Bluetooth and cordless telephones.

b) $802.11 \mathrm{a}$ (Wi-Fi $5.2 \mathrm{GHz}$ ) operates in the $5.2 \mathrm{GHz}$ band, which at this point is relatively free of interference from other electrical devices operating in this band.

WPANs are defined with IEEE standard 802.15 [13]. The most relevant enabling technologies for $\mathrm{m}$-Health systems are Bluetooth [14] and ZigBee [15]. Bluetooth technology was originally proposed by Ericsson in 1994, as an alternative to cables that linked mobile phone accessories. It is a wireless technology that enables any electrical device to communicate in the 2.5-GHz ISM (license free) frequency band. It allows devices such as mobile phones, headsets, personal digital assistants (PDAs), and portable computers to communicate and send data to each other without the need for wires or cables to link the devices together. It has been specifically designed as a low-cost, low-size, and low-power radio technology, which is particularly suited to the short range personal area network (PAN). The main features of Bluetooth are:

a) Real-time data transfer usually possible between $10-100 \mathrm{~m}$.

b) Supports both point-to-point wireless connections without cables between mobile phones and personal computers, as well as point-to-multipoint connections to enable ad hoc local wireless networks.

c) $400 \mathrm{~kb} / \mathrm{s}$ of data symmetrically or $700-150 \mathrm{~kb} / \mathrm{s}$ of data asymmetrically.

ZigBee (IEEE 802.15.4 standard) has been developed as a low data rate solution with multimonth to multiyear battery life and very low complexity. It is intended to operate in an unlicensed international frequency band. Potential applications include home automation, industrial control, and personal health care. The standard uses 16 channels at $2.4 \mathrm{GHz}$, ten channels at 902-928 MHz, and one channel at 868-870 MHz. The maximum data rates for each band are 250,40 , and $20 \mathrm{~kb} / \mathrm{s}$, respectively. The 2.4-GHz band operates worldwide while the sub-1-GHz band operates in North America, Europe, and Australia/New Zealand.

The recent surge research of mobile ad hoc networking will also trigger a parallel research activity in the application of these emerging technologies for m-Health.

3) Beyond $3 G$ Technologies and the Fourth-Generation (4G) Vision: It is expected that $4 \mathrm{G}$ will integrate existing wireless technologies including UMTS, GSM, wireless LAN, Bluetooth, ZigBee, Ultrawideband, and other newly developed wireless technologies into a seamless system. Some expected key features of $4 \mathrm{G}$ networks are stated as follows: a) High usability. 4G networks are all IP-based heterogeneous networks that allow users to use any system at anytime and anywhere. Users carrying an integrated terminal can use a wide range of application services provided by multiple wireless networks.

b) Support for multimedia services at low transmission cost. $4 \mathrm{G}$ provides for multimedia services with high data rate, good reliability, and at low per-bit transmission cost.

c) $4 \mathrm{G}$ provides personalized services, in order to meet the demands of different users for different services.

d) 4G systems also provide facilities for integrating services. Users can use multiple services from any service provider at the same time.

The main technological characteristics of $4 \mathrm{G}$ systems are expected to be as follows:

a) transmission speeds higher than in $3 \mathrm{G}(\min 50-100 \mathrm{Mb} / \mathrm{s}$, average $200 \mathrm{Mb} / \mathrm{s}$ );

b) system capacity larger than in $3 \mathrm{G}$ by ten times;

c) transmission costs per bit $1 / 10$ to $1 / 100$ of that of $3 \mathrm{G}$;

d) support for Internet protocols (IPv6);

e) various quality of service (QoS) providing many kinds of best effort multimedia services corresponding to users demand;

f) user-friendly services where users can access many services in a short time span as compared with other wireless systems of longer waiting times for response.

$4 \mathrm{G}$ advances will provide both mobile patients and citizens the choices that will fit their lifestyle and make easier for them to interactively get the medical attention and advice they need. When and where is required and how they want it regardless of any geographical barriers or mobility constraints. The concept of including high-speed data and other services integrated with voice services is emerging as one of the main points of future telecommunication and multimedia priorities with the relevant benefits to citizen-centered health-care systems. These creative methodologies will support the development of new and effective medical care delivery systems into the 21 st Century. The new wireless technologies will allow both physicians and patients to roam freely, while maintaining access to critical patient data and medical knowledge.

\section{B. Sensors for m-Health Systems}

With the aid of medical sensor technologies, m-Health can offer health-care services far beyond what the traditional telemedical systems (e.g., teleconsultation and teleconference) can possibly provide. A proper integration of medical sensors into m-Health systems would allow physicians to diagnose, monitor, and treat patients remotely without compromising standards of care.

Advances in new materials and signal processing research would enable the design of smart medical sensors to realize the real-time data recording and processing of multiphysiological signals. Many different kinds of medical sensors are now available on the market ranging from conventional sensors based on piezo-electrical materials for pressure measurements to infrared sensors for body temperature estimation and optoelectronic sensors monitoring $\mathrm{SpO}_{2}$, heart rate, $\mathrm{HRV}$, and blood pressure. 


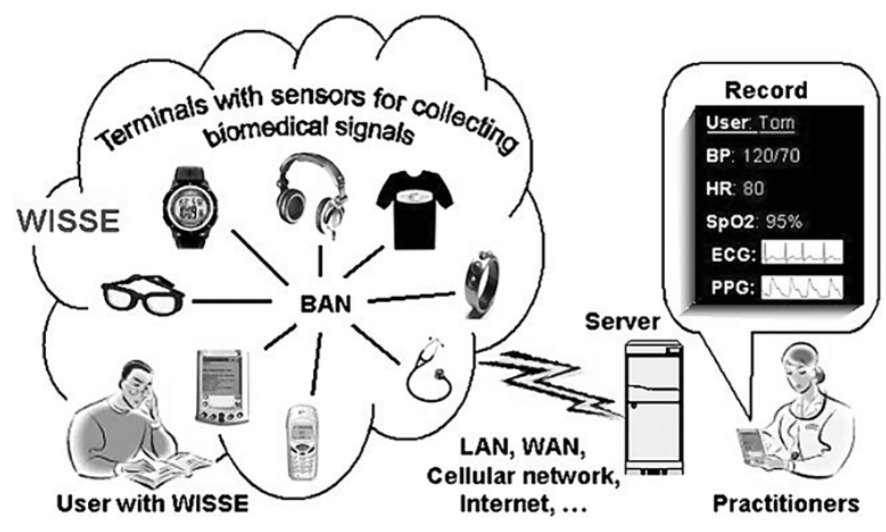

Fig. 2. WISSE: wearable devices with sensors and BAN for physiological data collection [16].

The rapid development in microelectronics and digital wireless technology makes it now possible to realize wireless medical sensors with networking capability to facilitate the joint processing of spatially and temporally collected physiological information from different parts of the body and the external communication for mobile health care. Such medical sensor networks greatly enhance the ability of physicians to timely examine and treat complex biological systems at a distance and effectively reduce the infrastructure cost at the hospital side and the travel expense at the patent end. m-Health equipped with medical sensors has the potential to transform the way health care is currently provided.

As sensor and computing technologies continue to evolve, their integration into wearable medical devices for the monitoring, diagnosis, and treatment of illnesses will become commonplace. To monitor human health constantly without disturbing users' normal daily activities, the wearable sensors and devices for physiological data collection should be designed to be so small that they will not affect the appearance and function of the user in which they are embedded. Miniature biomedical sensors and devices can also be embodied in or integrated with other wearable carriers (such as a finger ring), as shown in Fig. 2. This concept is being implemented in a project concerned with wearable intelligent sensors and systems for E-medicine (WISSE) with a body area network (BAN) forming the communication infrastructure [16], [17]. Operation of WISSE should be user-friendly, and require very little prior training, knowledge, and skills. Monitoring will be carried out actively but discreetly without the user or the people close by being aware of it. The ability of the terminals to communicate with each other will ensure a "one-stop-service," where measurements and data collected at different positions are centralized in a single location for user review and onward transmission to the external world. Wireless communication will be used for accessing health-care database wherever appropriate to allow free movement of the user [18], [19]. Last but not least, power consumption will also be taken into consideration in the overall design of wearable devices so that the frequency of recharging or replacement of batteries will not be a nuisance to the user. All of the above technical challenges must be solved in the development of wearable devices and systems in addition to the apparent issues of multisensor medical data fusion, system optimization, real-time wireless transmission, and information security.

Recent advances in microfabrication [20], integration of physical sensors [21], and new sensing [22] and stimulation technologies [20], [23] have the potential to revolutionize sensors and their integration, and create a new generation of sensor networks suitable for health monitoring applications. Ultimate examples include implanted sensors and implanted therapy devices such as drug infusion pumps [4].

\section{M-Health Systems}

M-Health systems are created as a synergy of emerging mobile medical computing, medical sensor technologies, and communication technologies. The trend within patient monitoring has been to allow the patient more mobility [24]. Possible medical applications include the following:

1) wearable systems and personal health-care monitoring [25], [26];

2) monitoring of soldiers in the battlefield [17];

3) emergency medical care and mass casualty event [27];

4) home monitoring [28]-[30];

5) computer-assisted rehabilitation and therapy [31];

6) social networking of relatives and peers of chronically ill patients.

Fig. 3 illustrates a generic system organization of m-Health systems [2]. The individual sensors are either wired to the personal monitoring system or connected wirelessly. A wearable healthmonitoring device using PAN or BAN can be integrated into the user's clothing. A typical example is GATECH Wearable Motherboard or Smart Shirt project [17]. Smart Shirt provides flexible data buses integrated into the fabric that is used to exchange information between sensors and the Smart Shirt controller. This system organization, however, is unsuitable for lengthy continuous monitoring when additional sensors could not be connected using Smart Shirt's data buses, such as monitoring of physical activity, electromyogram (EMG) of extremities, EEG, and other nonlocal signals. In that case, additional wiring significantly limits the system's unobtrusiveness, particularly during normal activity, such as intensive training or computer-assisted rehabilitation [31]. Technological advances in microtechnologies and nanotechnologies, application-specific integrated circuits, wireless networking, and embedded microcontrollers and radio interfaces on a single chip [32] have enabled wireless connectivity of individual intelligent sensors into a wireless body area network (WBAN) [33]. Individual sensors communicate with a personal server or a gateway using a custom wireless protocol [33], [45], Bluetooth [16], [42], or ZigBee [34]. The most critical parameter for sensor design and system integration is power consumption. Large power consumption increases battery capacity requirements, which increases battery size, and overall system size and weight. As a consequence, the user's acceptance is significantly reduced. However, existing computing platforms already feature extremely low power consumption, while recent research in the field of microelectromechanical systems resonators holds a promise of significant advances in low power communication and reduction in overall power consumption to microwatt range [35]. Reduced power consumption will 


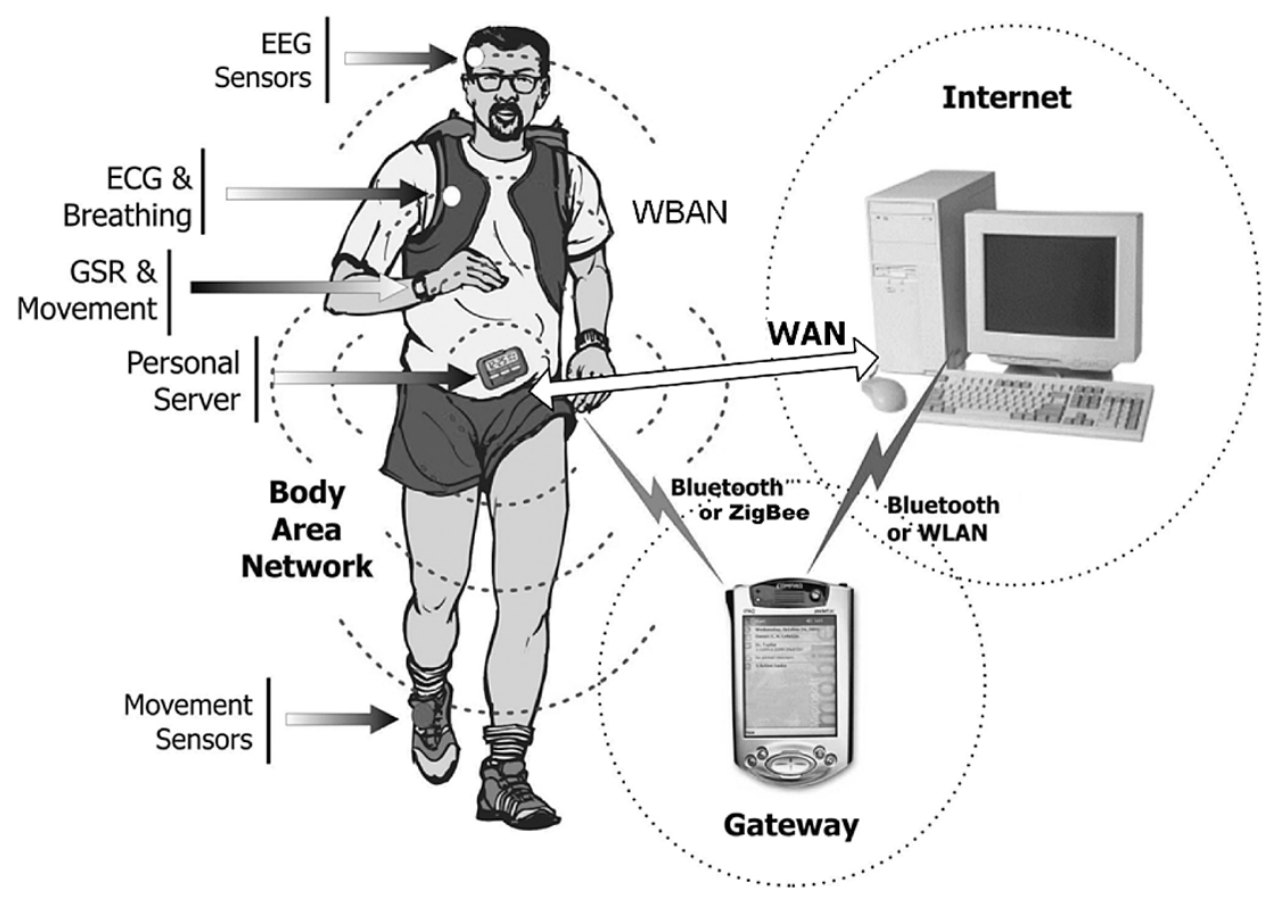

Fig. 3. Typical system architecture of m-health monitoring systems [2].

make possible externally powered sensors [20], [32] or energy scavenging from the environment. The main advantage of these systems for patients is the unobtrusive prolonged ambulatory monitoring, which allows the improved quality of life and faster response in the case of emergencies or triage and treatment in the case of mass casualty events.

WBAN allows seamless system integration of various sensors. At the same time, small range (1-2 m) reduces power consumption requirements, which directly reduces battery size and extends battery life. The same approach could be applied to the communication system of the personal server. Internet connectivity of the personal server requires significant power in the order of watts [45]. Reduced power consumption could be achieved by employing mobile gateways [2] or using meshnetwork of peer nodes to reach gateway with the Internet access [27].

The advances in this domain are closely related if not correlated with recent advances in pervasive and ubiquitous computing systems for health care [26]. In the next few years, we will see some major advances in these areas that will underpin the recent developments in pervasive computing systems for health care toward a new generation of m-Health systems.

The broad research areas that address the development in emerging mobile and network technologies will also support the complex systems that are related to matching current healthcare processes and infrastructures against the relevant cost-benefit business models. These include relevant business issues, such as interaction, visualization, planning and management, creating, monitoring, collaboration, information extraction, and education/training. Work that will integrate and focus research in areas of biomedical signal processing, ubiquitous computing, and data mining will become more prevalent in future $\mathrm{m}$-Health research. These systems will ultimately create a comprehensive and successful m-Health environment that can establish a holistic health-care framework that works globally. The main goals will be as follows.

1) to adapt current health-care infrastructures and existing medical (legacy) technologies in order to support future $\mathrm{m}$-Health services that are reliable, resilient, and cost effective for personalized health care;

2) to address the issues that are beyond the classical healthcare disciplines and expand our understanding of the disease formation and correlating issues, such as genomics and the changing environments;

3 ) to increase the understanding of the effect of emerging health-care technologies on human life and the fair access of health-care delivery and services to the global population.

\section{Future Challenges IN m-Health Systems}

\section{A. Categorization of m-Health Systems}

The expected developments in m-Health applications can be categorized into the following forms.

1) Administrative Health-Care Wireless Connectivity. This will deal with all the nonfinancial transactions such as $\mathrm{m}$-prescriptions, electronic patient record, procedures, and other electronic appointments that will provide better and efficient patient care.

2) Patient and Health-Care Financial Connectivity. These will deal with the processing of all future micropayments and purchases, billing and other financial services that will be within the domain of the $\mathrm{m}$-Health services in the hospitals and health-care points of access.

3) Medical Connectivity. This is the most important area. It will include all the m-diagnostic applications, monitoring, and other on-the-move applications between the different health-care providers and their patients. 
Future challenges in m-Health systems can be better understood by taking into account some of the basic and potential benefits of such systems. These can be summarized as follows.

- Provision of rapid response to critical medical care regardless of geographic barriers. For example, severely injured patients can be managed locally and access to a trauma specialist obtained by wireless connectivity.

- Flexible and swift access to expert opinion and advice at the point of care without delay and better management of medical resources. Interactive medical consultation and communication links of medical images and video data.

- Increased empowerment and management of medical expertise especially in rural and under-served areas.

- Intelligent personal health monitoring system to generate early warnings for a variety of medical conditions.

- Swift medical care in emergencies and management of medical data in catastrophes or natural disasters where conventional communication links may be disrupted.

- Continuous health monitoring promoting healthy lifestyles.

- Synergy of information from individual sensors providing better insight into the physiological state and the type and level of activity.

\section{B. Next-Generation m-Health Systems}

It is evident that organizations and the delivery of health care are being underpinned by the advances in $\mathrm{m}$-Health technologies. These advances are giving rise to a range of reforms in the way in which some health-care services are currently delivered. In the near future, the increasing medical data traffic and demand from different clinical applications and mobile medical scenarios will be compatible with the data rates of current $3 \mathrm{G}$ systems. Specifically, in a society penetrated by $3 \mathrm{G}$ systems, home medical care and remote diagnosis will become common, check-up by specialists and prescription of drugs will be enabled at home and in under-populated areas based on high resolution image transmission technologies and remote surgery, and virtual hospitals with no resident doctors will be realized. Preventive medical care will also be emphasized: for individual health management, data will constantly be transmitted to the hospital through built-in sensor and monitoring systems, e.g., in the patient's watch, accessories, or other items worn daily, and results will be fed back to the patient.

However, it is well known that current health-care systems are stuck with the equation [36]: Current Organization + New Technology $=$ Expensive Current Organization. To change this equation to one that delivers also cost benefits requires that the whole system of care processes, roles, and responsibilities of team members, organizations, and structures, including technology, is scrutinized in order to find where changes can be made. Ways must be innovated to organize work and to deliver services. This includes the citizen/patient as an active participant together with the health-care team of specialized professionals [37].

Hence, the deployment of these new generation mobile and wireless technologies will face challenges in dimensions that can be named as technological, economical, and social.
Technological challenges include:

- user acceptance issues, such as lightweight implementation, long battery life or battery-less sensors, biocompatibility, maintainability, usability, and reliability;

- seamless and secure integration of increased amounts of data from recording sessions;

- smart medical sensor design integrating sensing, processing, communications, computing, and networking together into a reduced volume for wearable devices;

- protocols for wireless medical sensor networks;

- support for QoS in wireless medical sensor networks.

Economical issues include:

- proof-of-principle challenges as required by approval agencies, such as FDA;

- availability of physicians for monitoring/consulting, or development of new services for prolonged monitoring in m-Health systems;

- price of preventive care versus savings from early detection;

- standardization of protocols and interfaces that will significantly decrease overall cost;

- new business opportunities for cheap, small, and possibly disposable sensors.

Social issues include:

- health-care coverage and patient's participation and reimbursement;

- liability issues, particularly the cost of lawsuit abuse and fraudulent lawsuits;

- promotion of healthy lifestyles (diabetes, obesity, chronically ill patients);

— the advantage of social networking of peers and interested parties;

- privacy and security of patient's records and transmissions.

All these issues present significant challenges as well as research opportunities in the field of m-Health.

\section{SPecial Section}

The goal of this special section is to provide a collection of papers that will reflect the spectrum of the recent advances in $\mathrm{m}$-Health technologies and the role of the emerging mobile and network technologies in m-Health systems and applications. We received 28 papers in response to the call, and 12 papers were accepted for this special section. The papers cover three main areas: communication systems, sensors, and systems.

Communication systems are covered by three papers. The first one by Aydin et al. covers implementation of an implanted sensor within the BAN and presents a design and an analysis of a communication system that can be implanted or swallowed for diagnostic and monitoring purposes [38]. The paper presents the design, simulation, and implementation of a direct-sequence spread-spectrum system for real-time communication of physiological data such as temperature, $\mathrm{pH}$, conductivity, and oxygen concentration. The second paper by Galego et al. presents a 
WAN communication system using UMTS technology. They evaluate the joint transmission of voice, real-time video, ECG signals, and medical scans in a realistic cellular multiuser simulation environment [39]. The authors assess the feasibility of UMTS to provide different QoS requirements for a multimedia application, consisting of four data services. The third paper by Chu and Ganz, presents use of $3 \mathrm{G}$ networks for simultaneous transmission of video, medical images, and ECG signals. They describe a portable teletrauma system that assists health-care centers by providing simultaneous transmission of a patient's video, medical images, and ECG signals, required throughout the prehospital procedure [40]. The performance of the system is evaluated over commercially available $3 \mathrm{G}$ wireless cellular data service and real network conditions.

Integration of application-specific sensors to the existing communication infrastructure is the focus of the next two papers. Lin et al. describe integration using wireless LAN technology. They propose a mobile physiological monitoring system for intrahospital transport of patients [41]. The system that integrates PDA technology and wireless LAN technology can provide continuous and real-time monitoring of vital signs including heart rate, three-lead $\mathrm{ECG}$, and $\mathrm{SpO}_{2}$. The paper reports the results of clinical evaluation of system performance. The paper by Woodward et al. presents the case of WAN connectivity using GPRS mobile phones with Bluetooth interface for sensors. They describe the modular design of an interface and processor for the transmission of multichannel biomedical signals over a Bluetooth link to GPRS-based mobile cellular networks [42]. This design allows transmission of physiological signals from a patient directly to a hospital using an unmodified mobile telephone.

Robotic tele-echography offers great medical advantages for a clinical examination of a remotely located patient. The next two papers examine different aspects of a tele-ultrasound system using a light robot for holding and moving a real probe on a distant patient for remote examination and medical diagnosis. Delgorge et al. present a teleoperated mobile ultrasound scanner for real-time medical ultrasound imaging acquisition [43]. Canero et al. propose a user interface for the visualization of the ultrasound images [44].

Implementation of $\mathrm{m}$-Health systems, issues, and challenges of system integration are presented in two papers. In the first paper, Anliker et al. describe a wearable monitoring and alert system (AMON) [45]. The system combines the monitoring of several physiological parameters with an integrated mobile phone link into a single bracelet-like device worn by the user. The paper describes the basic concepts and implementation, and provides valuable insights into typical problems such as signal integrity, real-time processing, and other implementation issues. In addition, user satisfaction has been evaluated and is reported.

Stringent limitations of size and power for patient monitors significantly limit functionality that physicians expect from diagnostic devices. For example, most cardiologists are trained to diagnose patients using 12-lead ECG. This requirement is unfeasible for ambulatory monitoring, but a cardiologist may reject systems with unfamiliar outputs [34]. To resolve this problem, Hadzievski et al. present an original concept of a mobile device for real-time reconstruction of 12-lead clinical ECG from a portable three-lead wireless device with cell phone interface [46]. The paper presents system organization, hardware and software design, and the results of the clinical study of cardiac rehabilitation.

The emergence of PDAs as powerful processing and communication platforms changes the scope and utility of mobile computers. The next group of papers covers different aspects of use of PDAs for m-Health applications. Nomadic and collaborative work of physicians in the hospital naturally finds PDAs as a platform of choice. This is particularly the case for WLAN equipped PDAs. The transmission itself could be used to localize the user and provide context aware services. For example, it is very likely that the physician in a patient's room will access the patient's medical record or the latest laboratory results. Therefore, the physician would be pleased to have this information conveniently available in context-aware applications. Rodriguez et al. present an agent-based system with location-aware access to hospital information and services [47]. A PDA is not only used to access hospital information systems and services, but to adapt the type of services and user interfaces based on detected location of the user. The location is estimated using a back propagation neural network with radio-frequency signal strengths used as inputs. Existing systems can provide enough precision for context aware delivery of information and services. This approach can significantly improve the efficiency and user acceptance of mobile technologies for m-Health applications.

PDA can be also used as data acquisition, processing, and communication platform as described by Lin et al. [41] and Woodward et al. [42]. Both systems are used mostly for data collection and transmission. However, local signal processing can significantly reduce power consumption and connection time and increase autonomy and reliability of the monitoring system. In most cases, PDAs provide enough processing power for real-time data processing and analysis. Goñi et al. present a sophisticated system for real-time processing and beat and rhythm classification of ECGs on a PDA [48]. Real-time processing eliminates the need for demanding data transfers. The paper demonstrates the feasibility of PDA implementation of the proposed set of algorithms.

An increasing number of clinical applications are supported on PDA platforms. PDA with wireless connectivity also provides the possibility for automatic updates and uploads, synchronization, and database access. Bojovic and Bojic present a mobile prescription reference system [49], as a very important mobile application for physicians. In addition, the paper describes automatic database updates using wireless hospital infrastructure, and provides user feedback.

We wish to thank all the authors for their contributions and the reviewers of this special section.

We hope that the papers presented in this section will contribute to the roadmap of future m-Health systems and improved and effective health-care delivery systems. 
Due to technical reasons, five of the m-Health papers are printed in this issue. The remaining seven will appear in the March 2005 issue.

\author{
ROBERT S. H. ISTEPANIAN, Guest Editor \\ Kingston University \\ London, U.K.
EMIL JovANOV, Guest Editor
University of Alabama in Huntsville \\ Huntsville, AL 35899 USA
}

\section{Y. T. ZHANG, Guest Editor \\ Chinese University of Hong Kong (CUHK) Hong Kong, China}

\section{ACKNOWLEDGMENT}

The authors would like to thank Prof. N. Saranummi and Dr. M. van Gils (VTT Information Technology, Finland), P. deGroen (Mayo Clinic), Dr. A. Milenkovic (University of Alabama in Huntsville), Dr. S. Warren (Kansas State University), Dr. D. Raskovic (University of Alaska Fairbanks), Dr. P. Bonato (Spaulding Rehabilitation Hospital), Dr. G. Taibi (SmartWorlds), Dr. H. Wang and N. Prag (MINT Research Centre, Kingston University, London), and Prof. S. Laxminarayan, Idaho State University, Idaho.

\section{REFERENCES}

[1] R. S. H. Istepanian and S. Laxminaryan, "UNWIRED, the next generation of wireless and internetable telemedicine systems-editorial paper," IEEE Trans. Inform. Technol. Biomed., vol. 4, pp. 189-194, Sept. 2000.

[2] E. Jovanov, A. Lords, D. Raskovic, P. Cox, R. Adhami, and F. Andrasik, "Stress monitoring using a distributed wireless intelligent sensor system," IEEE Eng. Med. Biol. Mag., vol. 22, no. 3, pp. 49-55, May/June 2003.

[3] C. S. Pattichis, E. Kyriacou, S. Voskarides, M. S. Pattichis, R. S. H. Istepanian, and C. N. Schizas, "Wireless telemedicine systems: An overview," IEEE Antennas Propagat. Mag., vol. 44, no. 2, pp. 143-153, Apr. 2002.

[4] C. E. Webb, "Chip shots," IEEE Spectr., vol. 41, no. 10, pp. 48-53, Oct. 2004.

[5] T. F. Budinger, "Biomonitoring with wireless communications," Аnnu. Rev. Biomed. Eng., vol. 5, pp. 383-412, 2003.

[6] R. S. H. Istepanian and H. Wang, "Telemedicine in UK," in European Telemedicine Glossary of Concepts, Standards Technologies and Users, 5th ed, L. Beolchi, Ed. Brussels, Belgium: European Commission-Information Society Directorate-General, 2003, pp. 1159-1165.

[7] R. S. H. Istepanian and J. Lacal, "M-Health systems: Future directions," in Proc. 25th Annu. Int. Conf. IEEE Engineering Medicine and Biology, Cancun, Mexico, Sept. 2003, pp. 17-21.

[8] R. S. H. Istepanian, S. Laxminarayan, and C. Pattichis, Eds., M-Health: Emerging Mobile Health Systems. New York: Kluwer/Plenum, 2005, to be published.

[9] D. Wisely and P. Eardley, IP for 3G-Networking Technologies for Mobile Communications. New York: Wiley, 2002.

[10] J. S. Blogh and L. Hanzo, Third-generation Systems and Intelligent Wireless Networking: Smart Antennas and Adaptive Modulation. New York: Wiley, 2002.

[11] B. Bing, Wireless Local Area Networks: The New Wireless Revolution. New York: Wiley, 2002.

[12] K. Banitsas, R. S. H. Istepanian, and S. Tachkara, "Applications of medical wireless LAN (MedLAN) systems," Int. J. Health Marketing, vol. 2, no. 2, pp. 136-142, 2002.
[13] IEEE 802.15 Working Group for WPAN [Online]. Available: http://www.ieee802.org/15/

[14] Bluetooth [Online]. Available: http://www.bluetooth.org/

[15] ZigBee Alliance [Online]. Available: http://www.zigbee.org/

[16] Y. T. Zhang, C. C. Y. Poon, S. D. Bao, and K. C. Lui, "A wearable and cuffless device for the continuous measurement of arterial blood pressure," in Proc. Int. Conf. Ubiquitous Healthcare, Seoul, Korea, Oct. 27-28, 2004.

[17] S. Park and S. Jayaraman, "Enhancing the quality of life through wearable technology," IEEE Eng. Med. Biol. Mag., vol. 22, no. 3, pp. 41-48, May/Jun. 2003.

[18] K. Hung and Y. T. Zhang, "Implementation of a WAP-based telemedicine system for patient monitoring," IEEE Trans. Inform. Technol. Biomed., vol. 7, no. 2, pp. 101-107, June 2003.

[19] —, "Wireless Internet in Telemedicine," in Wireless Internet Handbook: Technologies Standards, and Applications, B. Furht and M. Ilyas, Eds. Boca Raton, FL: CRC, 2003, pp. 511-535.

[20] M. Ghovanloo and K. Najafi, "A BiCMOS wireless stimulator chip for micromachined stimulating microprobes," in Proc. 2nd Joint EMBS/BMES Conf., Houston, TX, Oct. 23-26, 2002.

[21] P. Valdastri, A. Menciassi, A. Arena, C. Caccamo, and P. Dario, "An implantable telemetry platform system for in vivo monitoring of physiological parameters," IEEE Trans. Inform. Technol. Biomed., vol. 8, no. 3, pp. 271-278, Sept. 2004.

[22] E. Thrush, O. Levi, W. Ha, G. Carey, L. J. Cook, J. Deich, S. J. Smith, W. E. Moerner, and J. S. Harris Jr, "Integrated semiconductor vertical-cavity surface-emitting lasers and PIN photodetectors for biomedical fluorescence sensing," IEEE J. Quantum Electron., vol. 40, pp. 491-498, May 2004.

[23] G. E. Loeb, F. J. R. Richmond, J. Singh, R. A. Peck, W. Tan, Q. Zou, and N. Sachs, "RF-powered BION's for stimulation and sensing," in Proc. 26th IEEE EMBS, San Francisco, CA, Sept. 2004, pp. 4182-4185.

[24] D. Raskovic, T. Martin, and E. Jovanov, "Medical monitoring applications for wearable computing," Comput. J., vol. 47, no. 4, pp. 495-504, July 2004.

[25] Special Issue on Wearable Technology, IEEE Eng. Med. Biol. Mag., vol. 22, no. 3, May/June 2003.

[26] Special Issue on Pervasive Healthcare, IEEE Trans. Inform. Technol. Biomed., vol. 8, no. 3, Sept. 2004.

[27] D. Malan, T. R. F. Fulford-Jones, M. Welsh, and S. Moulton, "CodeBlue: An Ad Hoc sensor network infrastructure for emergency medical care," in Proc. MobiSys 2004 Workshop Applications Mobile Embedded Systems (WAMES 2004), Boston, MA, June 2004, pp. 12-14.

[28] V. Stanford, "Using pervasive computing to deliver elder care," IEEE Pervasive Computing, vol. 1, pp. 10-13, Jan./Mar. 2002.

[29] I. Korhonen, J. Parkka, and M. van Gils, "Health monitoring in the home of the future," IEEE Eng. Med. Biol. Mag., vol. 22, no. 3, pp. 66-73, May/June 2003.

[30] S. Warren, "Beyond telemedicine: Infrastructures for intelligent home care technology," in Pre-ICADI Workshop Technology for Aging, Disability, and Independence, London, U.K., June 26-27, 2003.

[31] J. M. Winters, Y. Wang, and J. M. Winters, "Wearable sensors and telerehabilitation: Integrating intelligent telerehabilitation assistants with a model for optimizing home therapy," IEEE Eng. Med. Biol. Mag., vol. 22, no. 3, pp. 56-65, May/June 2003.

[32] Center for Wireless Integrated Microsystems (WIMS). [Online]. Available: http://www.wimserc.org/

[33] E. Jovanov, J. Price, D. Raskovic, K. Kavi, T. Martin, and R. Adhami, "Wireless personal area networks in telemedical environment," in Proc. 3rd Int. Conf. Information Technology in Biomedicine, 2000, (ITABITIS2000), pp. 22-27.

[34] J. Welch, F. Guilak, and S. D. Baker, "A wireless ECG smart sensor for broad application in life threatening event detection," in Proc. 26th IEEE EMBS, San Francisco, CA, Sept. 2004, pp. 3447-3449.

[35] B. P. Otis and M. Rabaey, "A 300- $\mu$ W 1.9-GHz CMOS oscillator utilizing micromachined resonators," IEEE J. Solid-State Circuits, vol. 38, pp. 1271-1274, July 2003.

[36] R. S. H. Istepanian, N. Philip, X. H. Wang, and S. Laxminarayn et al., "Non-Telephone healthcare: The role of 4G and emerging mobile systems for future m-Health systems," in Int. Congress Medical and Care Compunetics, L. Bos et al., Eds., 2004, pp. 465-471.

[37] N. Saranummi, "Citizen-centric value network of professionals: Wellbeing services @ work," in Proc. E-Challenges E-2004, Vienna, Austria, Oct. 2004. 
[38] N. Aydin, T. Arslan, and D. R. S. Cumming, "A direct sequence spreadspectrum communication system for integrated sensor microsystems: Theory, design, and analysis," IEEE Trans. Inform. Technol. Biomed., to be published.

[39] J. R. Gállego, Á Hernández-Solana, M. Canales, J. Lafuente, A. Valdovinos, and J. Fernández-Navajas, "Mobile emergency care over the 3G UMTS systemto," IEEE Trans. Inform. Technol. Biomed., to be published.

[40] Y. Chu and A. Ganz, "A mobile teletrauma system using 3G networks," IEEE Trans. Inform. Technol. Biomed., vol. 8, no. 4, pp. 456-462, Dec. 2004.

[41] Y.-H. Lin, Y.-Y. Chen, P. C.-I. Ko, I.-C. Jan, J.-M. Wong, and G.-J. Jan, "A wireless PDA-based physiological monitoring system for patient transport," IEEE Trans. Inform. Technol. Biomed., vol. 8, no. 4, pp. 439-447, 2004.

[42] B. Woodward and M. F. A. Rasid, "Bluetooth telemedicine processor for multi-channel biomedical signal transmission via mobile cellular networks," IEEE Trans. Inform. Technol. Biomed., to be published.

[43] C. Delgorge, F. Courreges, L. Al Bassit, C. Novales, C. Rosenberger, N. Smith-Guérin, C. Brù, R. Gilabert, M. Vannoni, G. Poisson, and P. Vieyres, "A tele-operated mobile ultrasound scanner using a light weight robot," IEEE Trans. Inform. Technol. Biomed., to be published.
[44] C. Cañero, N. Thomos, G. Triantafyllidis, and M. G. Strintzis, "Mobile tele-echography: User interface design," IEEE Trans. Inform. Technol. Biomed., to be published.

[45] U. Anliker, J. A. Ward, P. Lukowicz, G. Tröster, F. Dolveck, M. Baer F. Keita, E. Schenker, F. Catarsi, L. Coluccini, A. Belardinelli, D. Shklarski, M. Alon, E. Hirt, and M. Vuskovic, "AMON: A wearable multiparameter medical monitoring and alert system," IEEE Trans. Inform. Technol. Biomed., vol. 8, no. 4, pp. 415-427, Dec. 2004.

[46] L. Hadzievski, B. Bojovic, P. Belicev, V. Vukcevic, S. Pavlovic, Z. Vasiljevic-Pokracic, and M. Ostojic, "A novel mobile transtelephonic system with synthesized 12-lead ECG," IEEE Trans. Inform. Technol. Biomed., vol. 8, no. 4, pp. 428-438, Dec. 2004.

[47] M. Rodriguez, J. Favela, E. A. Martinez, and M. A. Muñoz, "Locationaware access to hospital information and services," IEEE Trans. Inform. Technol. Biomed., vol. 8, no. 4, pp. 448-455, Dec. 2004.

[48] A. Goñi, J. Rodríguez, and A. Illarramendi, "Classifying ECGs in an on-line monitoring system," IEEE Trans. Inform. Technol. Biomed., to be published.

[49] M. Bojovic and D. Bojic, "Mobile PDR: A mobile medical information system featuring update via Internet," IEEE Trans. Inform. Technol. Biomed., to be published.

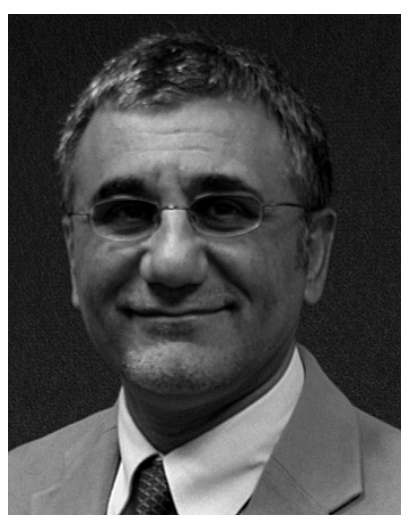

Robert S. H. Istepanian (M'94-SM'97) received the Ph.D. degree in electronic and electrical engineering from Loughborough University, U.K., in 1994.

From 1984 to 1998, he worked in different overseas industrial and academic positions. In 1988, he was a Visiting Research Fellow in the Department of Electronic and Electrical Engineering, Loughborough University, U.K. He was with the same department from 1994 to 1995 as a Postdoctoral Research Fellow. From 1996 to 1999, he was a Senior Lecturer at the University of Portsmouth, U.K. In 1999, he was an Associate Professor at the Universities of Western Ontario and Rycrson in Toronto, Canada. He was the head of the (Mobile Information Engineering and E-Med Systems) research group in the Department of Electronic and Computer Engineering Brunel University, West London, U.K., since 1999. In early 2003, he joined the School of Computing and Information Systems, Kingston University, London, U.K., as Professor of Data Communications and Founding Director of Mobile Information and Network Technologies (MINT) Research Centre.

Dr. Istepanian is the Recipient, Investigator, and Co-investigator of numerous research grants from the EU, EPSRC, and other industrially funded research projects. He has published more than 140 refereed journal and conference papers and three books, mostly in the areas of m-health and biomedical signal processing and communication systems. He is a Fellow of the Institute of Electrical Engineers. He also served on numerous technical committees for the IEEE, EMBS, and other international conferences. He currently serves on the editorial boards of the IEEE TRANSACTIONS ON INFORMATION TECHNOLOGY IN BIOMEDICINE and IEEE TRANSACTIONS ON NANOBIOSCIENCE, and was one of the founding special area editors of the IEEE TRANSACTIONS ON INFORMATION TECHNOLOGY IN BIOMEDICINE on Telemedicine and e-Health since 1997. He was the Guest Editor of IEEE TRANSACTIONS ON INFORMATION TECHNOLOGY IN BIOMEDicine, Special Issue on Mobile Telemedicine Systems, September 2000 and IEEE TRANSACtions on NANOBioscience, Special Issue on Microarray Imaging, published in December 2003. He is also an Associate Editor of the Journal of Mobile Multimedia and Journal on Information Technology in Healthcare. 


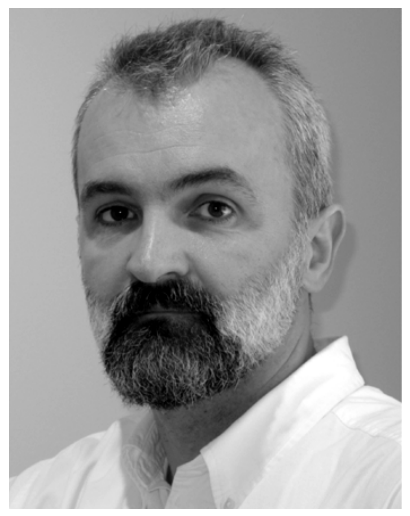

Emil Jovanov received the Dipl. Ing. M.Sc. and Ph.D. degrees in electrical and computer engineering from the University of Belgrade, Yugoslavia.

From 1984 to 1998, he worked in the "Mihajlo Pupin" Research Institute, Belgrade, Yugoslavia. He was an Adjunct Assistant Professor at the University of Belgrade between 1994 and 1998. In 1998, he joined the University of Alabama, Huntsville. He is currently an Associate Professor in the Electrical and Computer Engineering Department, University of Alabama, Huntsville. He has been developing wireless intelligent sensors for personal health monitoring and mobile computing for more than four years. He was principle Investigator or Co-investigator on several grants from NSF and industry in the field of wireless and sensor networks, wireless intelligent sensors, and wearable health monitors. He developed a distributed wireless heart rate monitoring system for Navy Aviation Medical Research Laboratory (NAMRL), Pensacola, FL. His research interests include ubiquitous and mobile computing, biomedical signal processing, and telemedical health monitoring, in which he has published several book chapters, over 20 journal papers, and more than 90 conference papers.

Dr. Jovanov serves as an Associate Editor of IEEE TRANSACTIONS ON INFORMATION TECHNOLOGY IN BIOMEDICINE and Applied Psychophysiology and Biofeedback.

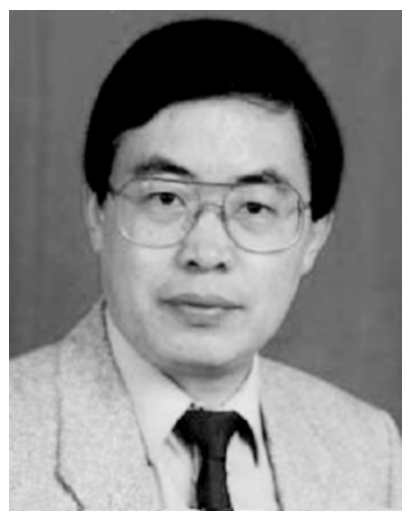

Y. T. Zhang received the Ph.D. degree from the University of New Brunswick, Canada, in 1990.

He was a Research Associate and Adjunct Assistant Professor at the University of Calgary, Canada, between 1989 and 1994. He joined the Chinese University of Hong Kong (CUHK) as a Lecturer in 1994, became an Associate Professor in 1996, and a Professor in 2002. He serves as the Director of Joint Research Centre for Biomedical Engineering. At CUHK, he has developed and teaches courses including biomedical modeling, medical instruments and sensors, and telemedicine techniques and applications. His research activities have focused on the development of biomodels and biosignal processing techniques to improve the performance of medical devices and biosensors, particularly for telemedicine. His work has been published in several books, over 20 scholarly journals, and numerous international conference proceedings. His current research interests include wearable medical devices and biosensors for mobile health, biological signal processing and modeling, telemedicine, and neural engineering.

Dr. Zhang has held various positions in professional organizations. He served the Technical Program Chair of the 20th Annual International Conference of the IEEE Engineering in Medicine and Biology (EMBS). He was the Chairman of the Biomedical Division of Hong Kong Institution of Engineers in 1996/1997 and 2000/2001. He was elected and served as an AdCom Member of IEEE-EMBS in 1999 and the Vice-President of the IEEE-EMBS in 2000 and 2001. He serves currently as an Associate Editor of IEEE TRANSACTIONS ON BIOMEDICAL ENGINEERING, an Associate Editor of IEEE TRANSACTIONS ON MobILE COMPUTING, and an Editorial Board Member for the Book Series of Biomedical Engineering, published by Wiley and IEEE Press. He is the General Conference Chair of the 27th Annual International Conference of IEEE-EMBS to be held in 2005. 